\title{
Anelídeos poliquetos da região de Ubatuba (SP) - padrões de distribuição geográfica
}

Eloisa H. Morgado

A. Cecilia Z. Amaral

\begin{abstract}
This work constitutes a systematic survey of the Annelida Polychaeta from the sublitoral of the Sâo Paulo State coast (Ubatuba), southeast Brazil. From 105 identified species, 20 are new records for the brazilian coast. An analysis of affinity degree between the fauna of the studied region and of the adjacents biogeographic provinces shows that 25,5\% of the species are endemic for the Paulista Province, 19,0\% amphi-american, 17,0\% cosmopolite and 16,8\% amphi-atlantic. An affinity with species from the Patagonian $(7,4 \%)$ and Caribbean $(2,0 \%)$ Provinces is smaller than that of other authors, for the southeast Brazilian coast.
\end{abstract}

\section{INTRODUÇÃO}

A determinação de padrões de distribuição assim como dos fatores responsáveis pelas amplas variações em densidade e produtividade de organismos marinhos têm sido considerados problemas fundamentais em ecologia marinha (DAY et al., 1971).

$\mathrm{O}$ conceito de que a temperatura constitui um fator limitante, essencial na distribuição, tem sido amplamente aceito. No entanto, fixar áreas limítrofes e distinguir conjuntos faunísticos com precisão, tem se mostrado extremamente difícil. Uma análise dos numerosos estudos sôbre a oceanografia do Atlân tico Sul e da costa brasileira, acompanhada de uma revisão cronológica da literatura sobre a zoogeografia dessa região, foi efetuada por PALÁCIO (1982).

No litoral do Estado de São Paulo, a região de Ubatuba é uma das mais estudadas, havendo um razoável número de trabalhos sobre a sistemática e a ecologia de anelídeos poliquetos de substrato mole: NONATO $(1958,1963,1966,1981)$, FORNERIS (1969), AMARAL (1977a - b, 1979, 1980a), ABREU (1978), LANA (1981, 1983), AMARAL \& NONATO $(1982,1984)$, BOLÍVAR \& LANA (1986), NONATO et al. (1986), AMARAL et al. (1987), BLANKENSTEIN \& LANA

1 Departamento de Zoologia, Instituto de Biologia - UNICAMP - C.P. 6109, 13801 Campinas, SP. 
(1987), LANA \& SOVIERZOSKI (1987), MORGADO (1988).

A biogeografia do grupo permanece no entanto, pouco conhecida. Padrões de distribuição geográfica da fauna de poliquetos do litoral brasileiro foram analisados por NONATO \& LUNA (1970b), ORENSANZ \& GIANUCA (1974), NONATO \& AMARAL (1976), AMARAL (1977b), TEMPERINI (1981), LANA (1984, 1987), MORGADO \& AMARAL (1986), BOLÍVAR \& LANA (1988) e MORGADO (1988). De uma maneira geral, tem sido observadas, principalmente na costa sudeste, altas taxas de endemismo e um elevado grau de afinidade com a fauna da Província Caribe ou Tropical.

Este trabalho representa um levantamento das espécies de anelídeos poliquetos de fundos sublitorais não consolidados, da região de Ubatuba (SP), compreendida entre as ilhas Anchieta e Vitória, incluindo uma análise dos padrões de distribuição geográfica da fauna regional e uma avaliação de seu grau de afinidade com a fauna das províncias biogeográficas adjacentes.

Levantamentos faunísticos regionais, com identificaçöes precisas das espécies são imprescindiveis para uma melhor compreensão da estrutura, funcionamento e variabilidade natural dessas comunidades, constituindo um requisito fundamental para análises de áreas sujeitas a perturbações ambientais e para o estabelecimento de programas de monitoramento costeiro.

\section{MATERIAL E MÉTODOS}

O material examinado provém de 6 estações fixas de coleta, estabelecidas entre as ilhas Anchieta e Vitória, e de um levantamento adicional da macrofauna epibentônica nas proximidades da Ilha Anchieta, município de Ubatuba, SP (Figura 1). As amostras foram obtidas de setembro de 1982 a setembro de 1983, utilizando-se um pegador do tipo Van Veen modificado $\left(0,05 \mathrm{~m}^{2}\right.$ de área) e uma draga retangular $(1 \mathrm{~cm}$ de malha).

A coleção estudada encontra-se integralmente depositada no Departamento de Zoologia, Instituto de Biologia, da Universidade Estadual de Campinas.

\section{ESPÉCIES, OCORRÊNCIA NA ÁREA ESTUDADA E DISTRIBUIÇĀO GEOGRÁFICA}

Um total de 105 espécies foram identificadas, sendo que duas delas foram mantidas a nível de família (Cirratulidae e Ctenodrilidae) e nove a nível genérico (Linopherus sp., Ninoe sp., Haploscoloplos sp., Scoloplos sp., Tharyx sp., Ophelina sp., Rhodine sp., Heteroclymene sp. e Praxillella sp.). Para ordenar as 35 famílias identificadas a nível específico foi adotado o critério taxonômico de HARTMAN (1959, 1965a). $\mathrm{Na}$ relação a seguir são apresentadas as referências essenciais à sinonímia e aquelas que se destacam pela presença de boas ilustrações, a ocorrência na área amostrada e a distribuição geográfica de cada espécie. 


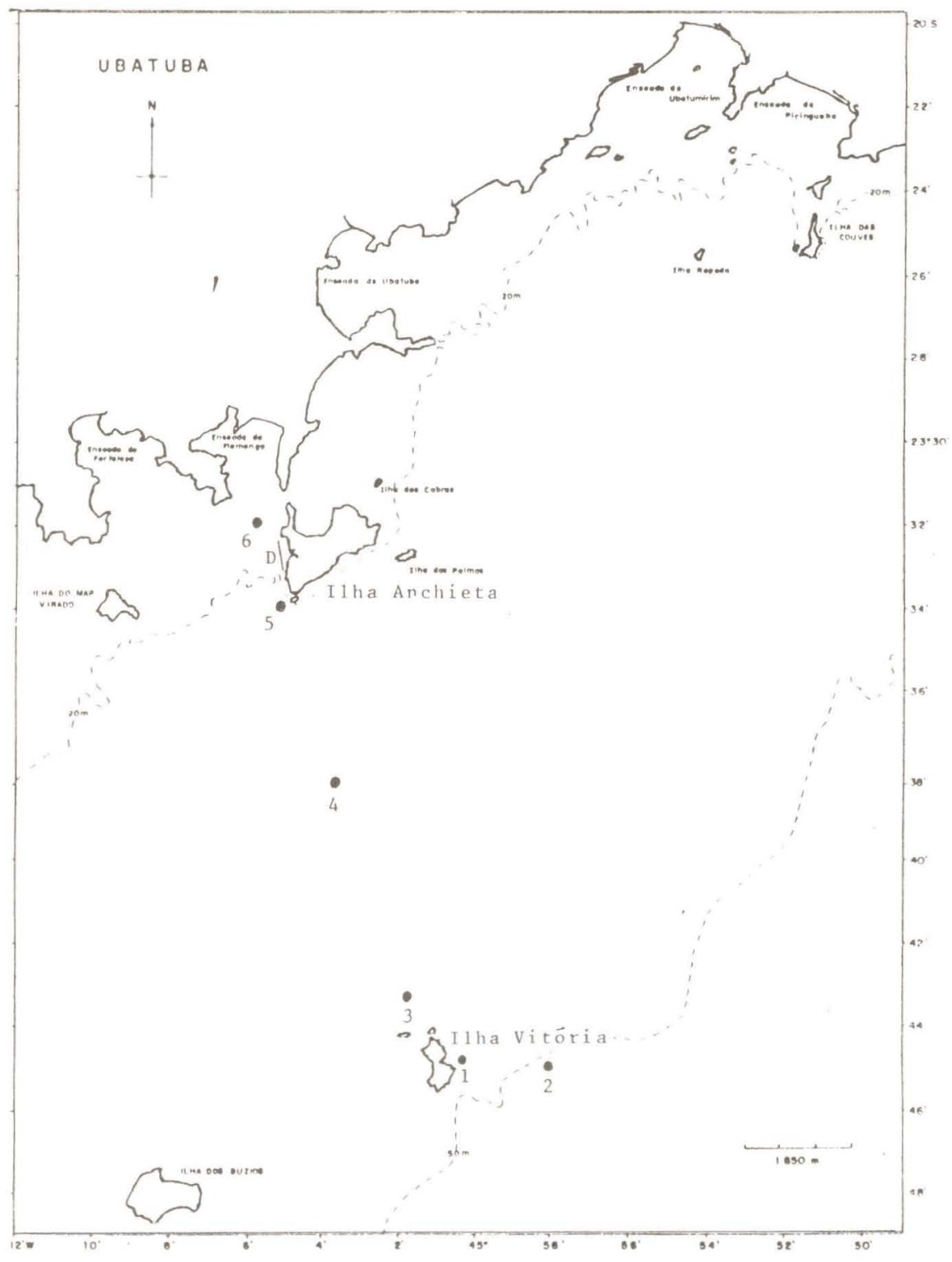

FIG. 1. Localização das Ilhas Anchieta e Vitória (SP), das estaçōes de coleta e percurso de dragagem (Estação 1: $23^{\circ} 44^{*} 08^{n} \mathrm{~S}-45^{\circ} 03^{n} \mathrm{~W}$; Estação 2: $23^{\circ} 45^{\circ} \mathrm{S}-44^{\circ}$ $58^{\prime} \mathrm{W}$; Estação 3: $23^{\circ} 43^{\prime} 03^{\prime \prime} \mathrm{S}-4^{\circ} 01^{\prime} 07^{\prime \prime} \mathrm{W}$; Estação 4: $23^{\circ} 38^{\prime \prime} \mathrm{S}-4^{\circ} 03^{\prime}$ $06^{\prime \prime} \mathrm{W}$; Estação 5: $23^{\circ} 34^{\prime} \mathrm{S}-4^{\circ} 05^{\circ} 01^{\prime \prime} \mathrm{W}$; Estação 6: $23^{\circ} 32^{\circ} \mathrm{S}-45^{\circ} 05^{\prime} 04^{\prime \prime} \mathrm{W}$; Dragagem (D): $45^{\circ} 05^{\circ} \mathrm{S}-23^{\circ} 33^{\prime} \mathrm{W}$. 


\section{POLYNOID AE}

Eunoe nodosa (Sars, 1861)

Polynoe nodosa Sars, 1861: 58.

Eunoe nodosa. - Fauvel, 1923: 51-52, fig. 18 a-e.

OCORRÊNCIA - Estação 5, em fundos de silte médio.

DISTRIBUIÇÃO - Mar do Norte, Canal da Mancha (Plymouth, Ilhas Inglesas); Atlântico Norte; Ártico.

Eunoe papillosa Amaral \& Nonato, 1982

Eunoe papillosa Amaral \& Nonato, 1982: 19, figs. 32-37.

OCORRÊNCIA - Dragagem.

DISTRIBUIÇÃO - Brasil: São Paulo (Ubatuba).

Eunoe serrata Amaral \& Nonato, 1982

Eunoe serrata Amaral \& Nonato, 1982: 19, figs. 38-44; Lana, 1984: 22 , figs. 12-13.

OCORRÊNCIA - Estações 2 e 6, em fundos de silte; dragagem.

DISTRIBUIÇÃO - Brasil: São paulo (Ubatuba) e Paraná.

\section{Harmothoe lunulata (delle Chiaje, 1841)}

Polynoe lunulata delle Chiaje, 1841 in Claparède, 1868: 378.

Harmothoe lunulata; Fauvel, 1923: 70, fig. 26 a-h; Day, 1967a: 71,

fig. 1.10 p-t; Rullier \& Amoureux, 1979: 149; Temperini, 1981:

6-7; Amaral \& Nonato, 1982: 22.

OCORRÊNCIA - Estação 5, em fundos de silte médio.

DISTRIBUIÇĀO - Costa Atlântica da Europa, do Mar do Norte ao Mediterrâneo; Oceano Índico (costa sul da Arábia); Pacífico americano (do oeste do Canadá ao sul da Califórnia); Golfo do Panamá; Ilhas Gálapagos. África do Sul. Atlântico sul ocidental (Uruguai e Argentina). Brasil: costa sudeste, Espírito Santo, Rio de Janeiro, São Paulo (Ubatuba e Santos) e Paraná. Esta ampla distribuição de Harmothoe lunulata permite considerå-la como espécie cosmopolita.

\section{SIGAIIONIDAE}

Sigalion cirriferum Orensanz \& Gianuca, 1974

Sigalion cirriferum Orensanz \& Gianuca, 1974: 4-6, figs. 2, 3 e 6. Sigalion taquari Amaral \& Nonato, 1984: 23, figs. 44-53.

OCORRÊNCIA - Estação 5, em fundos de areia fina e muito fina. DISTRIBUIÇÃO - Brasil: costa sul-sudeste, Rio de Janeiro (Ilha Grande) e São Paulo (Ubatuba); de Cassino (RS) até o Farol San Matias (Golfo San Matias, Argentina).

Sthenolepis grubei (Treadwell, 1901)

Sthenelais grubei Treadwell, 1901: 187-188.

Sthenolepis grubei; Nonato \& Luna, 1970a: 73, est. 4 figs. 37-45; 
Vol. 6(3), 1989

Amaral \& Nonato, 1984: 25-26.

OCORRÊNCIA - Dragagem.

DISTRIBUIÇÃO - Califórnia, Panamá, Equador e Porto Rico. Brasil: costa nordeste, Alagoas e sudeste, Rio de Janeiro (Ilha Grande) e São Paulo (Ubatuba).

Sthenolepis oculata (Hartman, 1942)

Leanira oculata Hartman, 1942a: 93-95, est. 8 figs. 1-5.

Sthenolepis oculata; Nonato \& Luna, 1970a: 73-74, est. 3 figs. 29-36;

Rullier \& Amoureux, 1979: 144; Temperini, 1981: 13; Amaral \& Nonato, 1984: 26.

OCORRÊNCIA - Estações 1 e 5, em fundos de silte grosso e médio; dragagem.

DISTRIBUIÇÃO - Pacífico oriental: costa da Califórnia, México, Guatemala e Equador. Mar das Antilhas (Cuba). Brasil: costa nordeste, Alagoas, e sul-sudeste, Bahia, Espírito Santo, Rio de Janeiro (Ilha Grande), Rio Grande do Sul (Torres).

\section{PHOLOIDAE}

Pholoe minuta (Fabricius, 1780)

Aphrodita minuta Fabricius, 1780: 514.

Pholoe minuta; Pettibone, 1963: 46, fig. 10 f-g; Day, 1967a: 100, fig. 1.18; Temperini, 1981: 10-11, figs. 23-28.

OCORRÊNCIA - Estações 2 e 6, em fundos de silte grosso e fino.

DISTRIBUIÇÃO - Ártico; Atlântico Norte: Islândia, da Noruega ao norte da França, do Labrador à Carolina do Norte; Mediterrâneo. Pacífico: do Mar de Bearing ao sul de Oregon, Chile, norte do Mar do Japão. África ocidental. Brasil: Espírito Santo, Rio de Janeiro, São Paulo e Paraná.

Pholoe synophthalmica Claparède, 1869

Pholoe synophthalmica Claparède, 1869: 79, est. 3 fig. 1; Fauvel, 1923: 120, fig. $44 \mathrm{i}-1$.

OCORRÊNCIA - Estações 1 e 4, em fundos de silte médio.

DISTRIBUIÇÃO - Canal da Mancha; Atlântico; Mediterrâneo.

Os exemplares examinados dessas duas espécies do gếnero Pholoe apresentaram características muito semelhantes a das descrições originais. No entanto, pequenas diferenças morfológicas observadas, talvez possam conferir a essas espécies o status de variedade novas para a costa brasileira.

\section{AMPHINOMIIDAE}

Linopherus ambigua (Monro, 1933)

Eurythoe ambigua Monro, 1933: 6, fig. 2.

Pseudoeurithoe ambigua; Hartman, 1945: 12; Gardiner, 1975: 103, 
fig. $5 \mathrm{n}-\mathrm{p}$; Nonato, 1981: 75 .

Linopherus ambigua; Fauchald, 1977: 12; Lana, 1984: 51-52.

OCORRËNCIA - Estação 6, em fundos de silte fino.

DISTRIBUIÇÃO - Costa pacífica do Panamá. Brasil: costa sudeste, Rio de Janeiro (Ilha Grande) e Säo Paulo (Ubatuba).

\section{PHYLODOCIDAE}

Anaitides mucosa (Oersted, 1843)

Phyllodoce (Anaitides) mucosa Oersted, 1843, fig. 1; Fauvel, 1923:

152-153, fig. 54 a-e; Pettibone, 1963: 81-82, fig. $18 \mathrm{f}-\mathrm{g}$.

Anaitides $\mathrm{cf}$. mucosa; Nonato, 1981: 76.

Phyllodoce cf. mucosa; Lana, 1984: 56, fig. 50.

OCORRÊNCIA - Dragagem.

DISTRIBUIÇÃO - Dinamarca, costa Atlântica: da Baía de Hudson até o Golfo do México; do Alasca até Califórnia e México; costa ocidental da África; Costa do Ouro. Brasil: costa sudeste, Rio de Janeiro (Ilha Grande) e Paraná.

Anaitides tamoya Nonato, 1981

Anaitides tamoya Nonato, 1981: 77-79.

OCORRÊNCIA - Dragagem.

DISTRIBUIÇÄO - Brasil: costa sudeste, Rio de Janeiro (Ilha Grande) e São Paulo (Ubatuba).

\section{HESIONIDAE}

Gyptis arenicolus (La Greca, 1946)

Oxydromus arenicolus La Greca, 1946: 273-276, 9 figs. OCORRÊNCIA - Estação 4, em fundos de silte médio. DISTRIBUIÇÄO - Golfo de Nápoles (Mergellina).

\section{Ophiodromus pugettensis (Johnson, 1901)}

Podarke pugettensis Johnson, 1901: 397-398, est. 3 figs. 23-25.

Ophiodromus pugettensis; Hessle, 1925: 20-21; Hartman, 1961: 67-68;

Imajima \& Hartman, 1964a: 83-84; Nonato, 1981: 85-87; Morgado

\& Amaral, 1984: 52-54, figs. 1-2.

OCORRÊNCIA - Estação 6, em fundos de silte fino.

DISTRIBUIÇÃO - Costa ocidental dos Estados Unidos e México; Peru; Japão; Brasil; costa sudeste, Rio de Janeiro (Ilha Grande) e São Paulo (Ubatuba).

\section{PILARGIDAE}

Cabira incerta Webster, 1879

Cabira incerta Webster, 1879: 279, est. 11 figs. 155-157; Pettibone, 1966: 178-179, fig. 11; Nonato, 1981: 88-89. 
OCORRÊNCIA - Estação 1, em fundos de silte grosso.

DISTRIBUIÇÃO - Costa leste dos Estados Unidos (Baía Chesapeake). Brasil: costa sudeste, Rio de Janeiro (Ilha Grande).

Loandalia americana minuta Nonato, 1981

Loandalia americana minuta Nonato, 1981: 89-90.

OCORRÊNCIA - Estações 1, 2, 3, 4 e 6, em fundos de areia fina e silte; dragagem.

DISTRIBUIÇÃO - Brasil: Costa sudeste, Rio de Janeiro (Ilha Grande) e São Paulo (Ubatuba, Santos e Cananéia).

Sigambra grubii Fritz Müller,1858

Sigambra grubii Fritz Müller, 1858: 214, est. 6 figs. 7-9; Pettibone, 1966: 182, fig. 13; Nonato, 1981: 90-93, figs. 97-98; Temperini, 1981: 18; Lana, 1984: 62-64, fig. 55.

OCORRÊNCIA - Nas 6 estações de coleta, em fundo de areia fina, areia muito fina e silte; dragagem.

DISTRIBUIÇÃO - Brasil: costa sul-sudeste, do Rio de Janeiro a Santa Catarina.

\section{NEREIDIDAE}

Ceratocephale oculata Banse, 1977

Ceratocephale loveni Malmgren, 1867: 61, est. 5 fig. 33; Pettibone, 1963: 152-154, fig. 42 a-b; Day, 1973: 38.

Ceratocephale oculata Banse, 1977: 620, fig 3; Gardiner \& Wilson, 1979: 165; Lana, 1984: 113-115.

OCORRÊNCIA - Dragagem.

DISTRIBUIÇÃO - Suécia, Noruega, Dinamarca, Iceland, Mar do Norte. Costa leste norte-americana (Carolina do Norte). Brasil: costa sudeste, Paraná.

Neanthes arenaceodonta Moore, 1903

Nereis (Neanthes) caudata della Chiaje, 1841: 96, 104, est. 102 figs. 10,15; Fauvel, 1923: 347-348, fig. 135 a-e; Hartmann-Schröeder, $1965 ; 123$.

Nereis arenaceodonta Moore, 1903: 720, est. 40 figs. 1-10.

Nereis (Neanthes) arenaceodonta; Pettibone, 1963: 162-165, fig. 44 i. OCORRÊNCIA - Estação 1, em fundos de silte médio. DISTRIBUIÇÃO - Cosmopolita.

Neanthes bruaca Lana \& Sovierzoski, 1987

Neanthes bruaca Lana \& Sovierzoski, 1987: 678-688, figs. 1-4. OCORRÊNCIA - Estação 1 e 6, em fundos de silte.

DISTRIBUIÇÃO - Brasil: costa sul-sudeste, São Paulo, Paraná e Santa Catarina. 
Nereis broa Lana \& Sovierzoski, 1987

Nereis broa Lana \& Sovierzoski, 1987: 683-688, figs. 5-8.

OCORRÊNCIA - Estações 1 e 5, em fundos de silte grosso e médio. DISTRIBUIÇÃO - Brasil: costa sudeste, São Paulo (Ubatuba).

\section{NEPHTYIDAE}

Aglaophamus juvenalis (Kinberg, 1866)

Aglaopheme juvenalis Kinberg, 1866a: 240.

Aglaophamus juvenalis; Hartman, 1948: 51, est. 7 fig. 1; Fauchald. 1976:

16 fig. 1 a-c; Lana, 1984: 86-88, figs. 78-79; Lana, 1986: 143-144, figs. 16-17.

OCORRÊNCIA - Estações 1 e 2, em fundos de silte grosso e médio; dragagem.

DISTRIBUIÇĀO - Brasil: Costa sudeste, Rio de Janeiro, São Paulo e Paraná.

Aglaophamus uruguayi Hartman, 1953

Aglaophamus uruguayi Hartman, 1953: 32, fig. 8 a-d; Lana, 1984: 88-89, figs. 80-81; Lana, 1986: 144, figs. 18-19.

OCORRÊNCIA - Estação 1, em fundos de silte grosso e médio.

DISTRIBUIÇÃO - Brasil: costa sul-sudeste, de São Paulo (Ubatuba) ao Rio Grande do Sul.

Nephtys acrochaeta Hartman, 1950

Nephtys acrochaeta Hartman, 1950: 114-116, est. 16 figs. 1-6; Lana, 1984: 77-79; Lana, 1986: 138-139, figs. 1-3. OCORRÊNCIA - Estação 2, em fundos de areia grossa.

DISTRIBUIÇÃO - Brasil: costa sul-sudeste, São Paulo, Paraná e Rio Grande do Sul.

Nephtys paradoxa Malm, 1874

Nephtys paradoxa Malm, 1874: 77, est. 1, figs. 2; Fauvel, 1923: 375, fig. 146. f-i; Hartman, 1950: 111.

OCORRÊNCIA - Estação 4, em fundos de areia fina e muito fina. DISTRIBUIÇĀO - Mar do Norte; Atlântico Norte. Ártico.

\section{GLYCERIDAE}

Glycera americana Leidy, 1855

Glycera americana Leidy, 1855: 147-148, est. 11 figs. 40-50; Hartman,

1950: 73-74; Pettibone, 1963: 213-215, fig. 54 a-e; Nonato \& Luna, 1970b: 71, fig. 26; Nonato, 1981: 103-104; Temperini, 1981: 28-29; Lana, 1984: 92-94, figs. 84-85.

OCORRÉNCIA - Estações 3 e 5, em fundos de areia fina; dragagem. DISTRIBUIÇÃO - Costa pacífica americana (do Canadá ao Peru); costa 
atlântica americana (de New England ao sudeste do Brasil). Brasil: costa sul-sudeste, Rio de Janeiro (llha Grande), São Paulo (Ubatuba) e Rio Grande do Sul.

\section{Glycera longipinnis Grube, 1878}

Glycera longipinnis Grube, 1878: 182, est. 8 fig. 9; Day, 1967a: 356, fig. 16.1 a-f; Nonato \& Luna, 1970b: 72-73, figs. 21-25. OCORRÊNCIA - Estação 2, em fundos de silte grosso.

DISTRIBUIÇÃO - Filipinas, Baía de Bengala, Golfo do Irã. África do Sul. Brasil: costa nordeste, Alagoas e Sergipe.

\section{Glycera oxycephala Ehlers, 1887}

Glycera oxycephala Ehlers, 1887: 121; Hartman. 1950: 70, figs. 3-4;

Gardiner, 1975: 163, fig. 18 h-j; Lana, 1984: 94-95, figs. 86-87. OCORRÊNCIA - Estação 5, em fundos de areia fina.

DISTRIBUIÇÄO - Ambos os lados da América tropical. Brasil: costa sudeste, São Paulo (Ubatuba) e Paraná.

\section{GONI ADID AE}

\section{Glycinde multidens Fritz Müller, 1858}

Glycinde multidens Fritz Müller, 1858: 214, figs. 4-6; Augener, 1918: 399-402, fig. 50; est. 3 fig 75; est. 7 fig. 196; Hartman, 1940: 249-251, est. 44 figs. 126-131; Hartman, 1950: 56; Nonato, 1981: 106-107, figs. 134-139; Lana, 1984: 104-105.

OCORRÊNCIA - Estações 1, 4 e 5, em fundos de areia muito fina, silte grosso e médio.

DISTRIBUIÇÃO - Brasil: costa sul-sudeste, Rio de Janeiro a Santa Catarina.

Goniada acicula Hartman, 1940

Goniada acicula Hartman, 1940: 252-254, est. 44 figs. 132-141; Hartman, 1950: 31-32, est. 4 figs. 2-7; Nonato, 1981: 110-112, figs. 140-143.

OCORRÊNCIA - Estações 3 e 4, em fundos de areia média.

DISTRIBUIÇÃO - Golfo da Califórnia; Colômbia, Venezuela e Jamaica; Brasil: costa sudeste, Rio de Janeiro (Ilha Grande) e São Paulo (Ubatuba).

\section{Goniada brunnea Treadwell, 1906}

Goniada brunnea Treadwell, 1906: 1174, figs. 67-70; Hartman, 1950: 17, est. 1 figs. 1-6; est. 4 fig. 1; Pettibone, 1963: 228, fig. 57 a-b; Lana, 1984: 102-103, figs. 97-99.

OCORRÊNCIA - Estações 3, 4 e 5, em fundos de areia fina e muito fina.

DISTRIBUIÇÃO - Costa pacífica norte-americana: Havaí; costa leste norte-americana (de New England à Carolina do Norte); Brasil: costa sudeste, Rio de Janeiro, São Paulo e Paraná. 
Goniada maculata Oersted, 1843

Goniada maculata Oersted, 1843: 33; Fauvel, 1923: 392-393, fig. 154 a-g; Hartman, 1950: 20, est. 1 figs. 7-8; Pettibone, 1963: 225-226, fig. 58 a-g; Day, 1967a: 367, fig. 16.4; Nonato, 1981: 112-113; Temperini, 1981: 29-30; Lana, 1984: 100-101, figs. 94-96. OCORRENCIA - Estações 2, 4 e 6, em fundos de silte; dragagem. DISTRIBUIÇÃO - Cosmopolita. Brasil: costa-sudeste, São Paulo, Paraná e Santa Catarina.

\section{ONUPHIDAE}

\section{Diopatra cuprea (Bosc, 1802)}

Nereis cuprea Bosc, 1802: 142.

Diopatra cuprea; Hartman, 1944: 54-55, est. 1 figs. 9-14; Pettibone, 1963: 250-254, fig. 66; Nonato \& Luna, 1970b: 73; Lana, 1984: 142-143, figs. $138-140$.

OCORRÊACIA - Estação 3, em fundos de areia grossa; dragagem.

DISTRIBUIÇÃO - Costa leste norte-americana (Massachusets e Flórida), Golfo do México; Antilhas; Panamá; costa oeste africana. Pacífico tropical. Brasil: costa nordeste, Alagoas e Sergipe; sudeste, São Paulo (Ubatuba).

\section{Diopatra tridentata Hartman, 1944}

Diopatra tridentata Hartman, 1944: 61-63, est. 2 figs. 37-43; est. 17 figs. 335-336; Nonato \& Luna, 1970b: 75; Gardiner, 1975: 185, fig. 23 j-n; Nonato, 1981: 116; Temperini, 1981: 30.

OCORRÊNCIA - Estações 1, 2, 3 e 6, em fundos de areia fina, silte médio e silte fino; dragagem.

DISTRIBUIÇÃO - Costa pacífica americana, da Califómia ao Equador; costa atlântica americana, da Carolina do Norte ao sul-sudeste do Brasil: Espírito Santo, Rio de Janeiro, São Paulo e Santa Catarina.

\section{Kinbergonuphis difficilis (Fauchald, 1982)}

Onuphis setosa; Orensanz, 1974a: 89-93, est. 4 e figs. $1-12$ e est. 5 figs. 1-12. A espécie $O$. setosa identificada por Orensanz, 1974a foi descrita por Kinberg, 1865: 560.

Onuphis (Onuphis) difficilis Fauchald, 1982a: 203, fig. 1. Kinbergonuphis difficilis (Fauchald, 1982b): 18, fig. 8a; Lana, 1984: 165-167.

OCORRÊNCIA - Estações 2, 4 e 5, em fundos de areia muito fina e silte médio.

DISTRIBUIÇÃO - Atlântico: Uruguai e Brasil: costa sudeste, São Paulo e Paraná.

Kinbergonuphis fauchaldi Lana, 1984

Kinbergonuphis fauchaldi Lana, 1984: 161-163, figs. 177-181. OCORRÊNCIA - Estaçöes 1, 3 e 4, em fundos de areia média, areia 
fina e silte médio.

DISTRIBUIÇÃO - Brasil: costa sudeste, Paraná.

Kinbergonuphis orensanzi (Fauchald, 1982)

Onuphis (Onuphis) orensanzi Fauchald, 1982a: 205, fig. 2.

Kinbergonuphis orensanzi (Fauchald, 1982b): 27, fig. 8b; Lana, 1984: 163-165, fig. 183 .

OCORRÊNCIA - Estação 5, em fundos de areia fina.

DISTRIBUIÇÃAO - Atlântico Sul: Brasil: costa sudeste, Paraná; Argentina (Rio da Prata).

Kinbergonuphis tenuis (Hansen, 1882)

Onuphis tenuis Hansen, 1882: 10; Orensanz, 1974a: 87-89, est. 3 figs. 1-11.

Kinbergonuphis tenuis; Fauchald, 1982b: 13-14, fig. 5c.

OCORRÊNCIA - Estações 2, 4 e 5, em fundos de areia fina, silte grosso e médio; dragagem.

DISTRIBUIÇÃO - Atlântico Sul: Brasil: costa sudeste, São Paulo; Uruguai.

\section{Mooreonuphis intermedia (Kinberg, 1865)}

Onuphis fragilis Kinberg, 1865: 561; Orensanz, 1974a: 94-95, est. 7 figs. 1-13.

Onuphis intermedia Kinberg, 1865: 560.

Mooreonuphis intermedia; Fauchald, 1982b: 60, fig 17e; Lana, 1984: 155-158, figs. 165-168.

OCORRÊNCIA - Estações 3, 4 e 5, em fundos de areia média, fina e muito fina.

DISTRIBUIÇÃO - Costa atlântica sul-americana; do sudeste do Brasil (Rio de Janeiro) ao Rio da Prata (Argentina).

Mooreonuphis nebulosa (Moore, 1911)

Onuphis nebulosa Moore, 1911: 269-273, est. 17 figs. 58-68; Hartman, 1944: 75 , est. 4 figs. 76-85.

Mooreonuphis nebulosa; Fauchald, 1982b: 56-57, fig. 17a.

OCORRÊNCIA - Dragagem.

DISTRIBUIÇÃO - Ambos os lados da América. Brasil: costa sudeste, Rio de Janeiro (Ilha Grande) e São Paulo (Ubatuba).

Mooreonuphis pallidula (Hartman, 1965)

Nothria pallidula Hartman, 1965b: 105-106, est. 17 d-h.

Mooreonuphis pallidula; Fauchald, 1982b: 62, fig. $17 \mathrm{~b}$.

OCORRÊNCIA - Estação 4, em fundos de areia muito fina.

DISTRIBUIÇÃO - Costa atlântica dos Estados Unidos.

Onuphis dibranchiata Willey, 1905

Onuphis dibranchiata Willey, 1905: 277-278, est. 4 e fig. 100; Fauchald, 1982b: 44-45. 
OCORRÊNCIA - Dragagem.

DISTRIBUIÇÃO - Oceano Índico

Marphysa kinbergi Mc Intosh, 1910

Marphysa kinhergi Mc Intosh, 1910: 451, est. 74 fig. 9; Orensanz, 1975: 103, est. 6 figs. 1-9; Temperini, 1981: 37-38, figs. 80-85; Lana, 1984: 185-187, figs. 199-200.

OCORRÊECIA - Estação 2, em fundos de silte médio.

DISTRIBUIÇÃO - Atlântico: Cabo Finisterre, Golfo da Guiné e Uruguai. Brasil: costa sudeste, São Paulo e Paraná.

\section{EUNICIDAE}

Eunice vittata (delle Chiaje, 1828)

Nereis vittata delle Chiaje, 1828: 195.

Eunice vittata; Fauvel, 1923: 404, fig.158 h-n; Hartman, 1944: 118; Day, 1967a: 385, fig. 17.3 a-e; Hartman, 1968: 721, figs. 1-5; Fauchald, 1970: 48, est. 3 figs. 1-m; Nonato \& Luna; 1970b: 82; Temperini, 1981: 36-37; Lana, 1984: 181-183.

OCORRÊNCIA - Estação 6, em fundos de silte grosso; dragagem. DISTRIBUIÇÃO - Atlântico Norte, de New England à Carolina do Norte, África Ocidental, Mediterrâneo. Pacífico; Califórnia do Sul; Japão. Brasil: costa nordeste, Alagoas e sul-sudeste, São Paulo (Ubatuba), Paraná e Rio Grande do Sul.

\section{LUMBRINERIDAE}

Augeneria tentaculata Monro, 1930

Augeneria tentaculata Monro, 1930: 140, fig. 52 a-k; Day, 1967a: 430, fig. 17.14 h-i; Orensanz, 1973: 369-371, est. 11 figs. 1-8.

OCORRÊNCIA - Estações 1, 2, 3 e 5, em fundos de areia fina, silte grosso e silte médio.

DISTRIBUIÇÃO - Uruguai e Província de Buenos Aires: ao norte das Malvinas; Ilhas Orcadas do Sul; Arquipélago de Palmer.

\section{Lumbrineris angrense Nonato, 1981}

Lumbrineris angrense Nonato, 1981: 132-134.

OCORRÊNCIA - Estações 4 e 5 , em fundos de areia média e fina.

DISTRIBUIÇÃO - Brasil: costa sudeste, Rio de Janeiro (Ilha Grande) e São Paulo (Ubatuba).

Ninoe brasiliensis Kinberg, 1865

Ninoe brasiliensis Kinberg, 1865: 567; Orensanz, 1973: 381-382, est. 14, figs. 1-7; Nonato, 1981: 140-143, fig. 163; Temperini, 1981: 39-40; Lana, 1984: 168-169, fig. 185.

OCORRÊENCIA - Estações 1, 2, 3, 4 e 5, em fundos de areia fina, 
areia muito fina, silte grosso e silte médio.

DISTRIBUIÇĀO - Atlântico Sul: da costa sudeste do Brasil (Rio de Janeiro) à Argentina (Puerto Quenquém).

\section{ARABEIUDAE}

\section{Drilonereis filum (Claparède, 1868)}

Lumbriconereis filum Claparède, 1868: 454, est. 9 fig. 1.

Drilonereis filum; Fauvel, 1923: 436, fig. 174 a-h; Hartman, 1968:

799, 2 figs.; Fauchald, 1970: 136, est. 21 fig h; Orensanz, 1974b: 395-397, est. 6 figs. 1-5; Lana, 1984: 193-194, figs. 210-211.

OCORRẼNCIA - Estação 5, em fundos de areia fina.

DISTRIBUIÇÃO - Cosmopolita. Brasil: costa sudeste, São Paulo (Ubatuba).

\section{Labrorostratus prolificus Amaral, 1977}

Labrorostratus prolificus Amaral 1977 a: 285-292, 8 figs.

OCORRÊNCIA - Estações 1 e 4, em fundos de areia média e silte grosso.

DISTRIBUIÇÃO - Brasil: costa sudeste, São Paulo (Ubatuba).

\section{Notocirrus lorum Ehlers, 1897}

Notocirrus lorum Ehlers, 1897: 78-80, est. 5 fig. 125-128; Hartman, 1944: 175; Orensanz, 1974b: 393-394, est. 5 figs. 1-4; Nonato, 1981: $145-146$.

OCORRÊNCIA - Estações 3, 4 e 5, em fundos de areia média, areia fina e silte médio.

DISTRIBUIÇĀO - Perú; Chile; Brasil: costa sudeste, Rio de Janeiro (Ilha Grande) e São Paulo (Ubatuba).

\section{DORVILLIDAE}

Stauronereis annulata Moore, 1906

Stauronereis annulata Moore, 1906: 225-227, est. 10 figs. 12-13, est 11 figs. 18-22.

Dorvillea annulata; Fauchald, 1970: 152-154, est, 25 figs. a-j.

OCORRÊNCIA - Estação 4, em fundos de silte médio.

DISTRIBUIÇÃO - Washington, Western México (próximo à Ilha Cedros e Golfo da Califórnia).

\section{ORBINIIDAE}

Scoloplos (Leodamas) ohlini (Ehlers, 1901)

Aricia ohlini Ehlers, 1901: 167-169, est. 21 figs. 9-13.

Scoloplos (Leodamas) ohlini; Hartman, 1957: 287-289, est. 31 figs. 
6-8; Hartman, 1966: 11-13, est. 2 fig. 8; Nonato, 1981: 157-159. OCORRÊNCIA - Estação 1, em fundos de silte médio.

DISTRIBUIÇÃO - Baixa Califórnia; México ocidental; Brasil: costa norte e sudeste, Rio de Janeiro (Ilha Grande).

\section{Scoloplos (Leodamas) rubra (Webster, 1879)}

Aricia rubra Webster, 1879: 253-255, est. 9 figs. 123-126.

Scoloplos (Leodamas) rubra; Hartman, 1951: 74; Hartman, 1957: 291, fig. 32; Nonato, 1981: 158-159.

OCORRÊNCIA - Dragagem.

DISTRIBUIÇÃO - Costa atlântica dos Estados Unidos (da Virgínia ao Golfo do México). Brasil: costa sudeste, Rio de Janeiro (Ilha Grande) e São Paulo (Ubatuba).

\section{Scoloplos treadwelli Eisig, 1914}

Scoloplos treadwelli Eisig, 1914: 405-407; Augener, 1933: 318; Hartman, 1957: 283-284; Nonato, 1981: 160-162, fig. 181. OCORRÊNCIA - Estações 2, 5 e 6, em fundos de silte.

DISTRIBUIÇÃO - Descrita originalmente para Porto Rico a espécie foi também assinalada na Califórnia, México e Jamaica. Brasil: costa sudeste, Rio de Janeiro (Iha Grande) e São Paulo (Ubatuba).

\section{PARAONIDAE}

\section{Aricidea neosuecica Hartman, 1965}

Aricidea suecica Eliason, 1920: 52-55, figs. 14-15. Aricidea near suecica; Hartman, 1957: 319-320, est. 43 fig. 7. Aricidea neosuecica Hartman, 1965b: 137; Hartman, 1969: 63, 1 fig. OCORRÊNCIA - Estação 1, em fundos de silte grosso.

DISTRIBUIÇÃO - Costa americana: sul da Califórnia; New England.

\section{Cirrophorus lyriformes (Annenkova, 1934)}

Paraonis (Paraonides) lyriformis Annenkova, 1934: 656-659, fig. 1b. Aricidea (Cirrophorus) furcata Hartman, 1957: 324-325, est. 43 fig. 6. Cirrophorus lyriformes; Hartman, 1965b: 138.

Cirrophorus furcatus; Hartman, 1969: 69, 1 fig.; Nonato, 1981: 163-164.

Hartman (1969) consideraA. (Cirrophorus) furcata Hartman, 1957, como sendo sinônimo de $C$. furcatus.

OCORRÊNCIA - Estações 1, 5 e 6, em fundos de silte.

DISTRIBUIÇÃO - Costa ocidental e oriental dos Estados Unidos. Brasil: costa sudeste, Rio de Janeiro (Ilha Grande).

\section{SPIONIDAE}

Laonice branchiata Nonato, Bolívar \& Lana, 1986

Laonice branchiata Nonato, Bolívar \& Lana, 1986: 21-24, figs. 1-7. OCORRÊNCIA - Estações 1, 4 e 6, em fundos de silte grosso e médio; 
dragagem.

DISTRIBUIÇÃO - Brasil: costa sudeste, Rio de Janeiro, São Paulo e Paraná.

\section{Laonice cirrata (Sars, 1851)}

Nerine cirrata Sars, 1851: 207.

Laonice cirrata; Malmgren, 1867: 200; Fauvel, 1927: 38, fig. 12 a-e; Hartman, 1966: 16-17, est. 3 figs. 13-15; Foster, 1971: 69-71, figs. 155-160; Bolívar, 1986: 18-20, figs. 27-31.

OCORRÊENCIA - Estações 2 e 3, em fundos de areia média e silte fino; dragagem.

DISTRIBUIÇÃO - Cosmopolita. Brasil: costa sudeste, Paraná.

\section{Paraprionospio pinnata (Ehlers, 1901)}

Prionospio pinnata Ehlers, 1901: 163; Kirkegaard, 1959: 22; Hartman \& Barnard, 1960: 114, est. 9 figs. 1-5; Imajima \& Hartman, 1964b: 286; Hartman-Schröeder, 1965: 211; Banse \& Hobson, 1968: 29.

Prionospio africana Augener, 1918: 402-405, fig. 51, est. 6, figs. 162-163.

Paraprionospio pinnata; Foster, 1971: 102-104, figs. 237-246; Bolívar, 1986: 35-36, figs. 73-79.

Paraprionospio africana; Nonato, 1981: 166-169.

OCORRÊNCIA - Estações 1, 4, 5 e 6, em fundos de areia muito fina e silte; dragagem.

DISTRIBUIÇÃO - Atlântico; Carolina do Norte e Marrocos até o sul da África; Golfo do México; Caribe; Índico; Pacífico; oeste do Canadá ao Chile, Japão, Nova Zelândia. Brasil: costa sudeste, São Paulo (Ubatuba, Santos e Cananéia) e Paraná.

\section{Prionospio malmgreni Claparède, 1870}

Prionospio malmgreni Claparède, 1870; Fauvel, 1927: 61-62, fig. 21 a-e; Hartman, 1969: 159, 4 figs.

OCORRÊENCIA - Estações 3 e 5, em fundos de areia média e silte médio.

DISTRIBUIÇÃO - Atlântico; da Carolina do Norte e Mar do Norte até Ilha da Madeira; Mediterrâneo; Japão; África do Sul. Brasil: costa sudeste, Rio de Janeiro (Ilha Grande) e São Paulo (Ubatuba).

\section{Spiophanes missionensis Hartman, 1941}

Spiophanes missionensis Hartman, 1941: 296-298, est. 46 figs. 17-21; Hartman, 1969: 185, 4 figs.; Bolívar, 1986: 32-33, figs. 62-66. Spiophanes cf. missionensis; Nonato, 1981: 169:170.

OCORRÊNCIA - Estações 1, 4 e 5, em fundos de areia muito fina, silte grosso e silte fino.

DISTRIBUIÇÃO - Pacífico; sul da Califórnia. Atlântico; Brasil: costa sudeste, Rio de Janeiro (Ilha Grande) e São Paulo (Santos e Ubatuba) e Paraná. 


\section{MAGELONIDAE}

Magelona nonatoi Bolívar \& Lana. 1986

Magelona nonatoi Bolívar \& Lana, 1986: 142-144, figs. 31-42.

OCORRÊNCIA - Estações 2 e 5, em fundos de silte médio e grosso; dragagem.

DISTRIBUIÇÃO - Brasil: costa sudeste, Rio de Janeiro e São Paulo.

\section{Magelona variolamellata Bolívar \& Lana, 1986}

Magelona variolamellata Bolívar \& Lana, 1986: 137-140, figs. 13-28. OCORRÊNCIA - Estações 2, 4 e 6, em fundos de silte grosso e médio. DISTRIBUIÇÃO - Brasil: costa sudeste, Rio de Janeiro, São Paulo e Paraná.

\section{POECILOCHAETIDAE}

Poecilochaetus australis Nonato, 1963

Poecilochaetus australis Nonato, 1963: 17-26, figs. 1-4 e figs. 1-11; Orensanz \& Gianuca, 1974: 16. OCORRÊNCIA - Estações 5 e 6, em fundos de areia muito fina e silte grosso; dragagem.

DISTRIBUIÇÃO - Brasil: costa sul-sudeste, São Paulo (Santos e Ubatuba) e Rio Grande do Sul (Rio Grande).

\section{CIRRATULIDAE}

Cirratulus filiformis Keferstein, 1862

Cirratulus filiformis Keferstein, 1862: 122, est. 10 figs. 28-31; Fauvel, 1927: 94, fig. 33h; Day, 1967b: 511, fig. 20.3 e-f. OCORRÊNCIA - Estação 1, em fundos de sil te médio. DISTRIBUIÇÃO - Atlântico leste; Canal da Mancha, Suécia, Escócia, Marrocos; Senegal; oeste da África tropical; Golfo Pérsico. Brasil: costa sudeste, São Paulo (Ubatuba).

\section{CHAETOPTERIDAE}

Chaetopterus variopedatus (Renier, 1804)

Tricoelia variopedatus Renier, 1804: 18

Chaetopterus variopedatus; Fauvel, 1927: 77-79, fig. 26 a-n; Day, 1967b: 529, fig. 22.2 a-g; Hartman, 1969: 209, 3 figs.; Rullier \& Amoureux, 1979: 180; Nonato, 1981: 172-173. OCORRÊNCIA - Dragagem.

DISTRIBUIÇÃO - Cosmopolita. Na costa brasileira ocorre desde o nordeste (Alagoas) até o Rio Grande do Sul. 


\section{FLABEULIGERIDAE}

Diplocirrus glaucus var. australis Nonato, 1981

Diplocirrus glaucus var. australis Nonato, 1981: 175-177.

OCORRÊNCIA - Dragagem.

DISTRIBUIÇÄO - Brasil: costa sudeste, Rio de Janeiro (Ilha Grande).

\section{Pherusa laevis (Stimpson, 1856)}

Siphonostomun laeve Stimpson, 1856: 391

Pherusa laevis; Augener, 1918: 430, fig. 40; Day, 1967b: 661-662, Fig. $32.3 \mathrm{a}-\mathrm{c}$.

OCORRÊNCIA - Estações 2, 3 e 4, em fundos de areia grossa, média e fina.

DISTRIBUIÇÃO - Costa sudoeste da África.

\section{Piromis eruca (Claparède, 1869)}

Trophonia eruca Claparède, 1869: 105, est. 15 fig. 2.

Stylarioides eruca; Fauvel, 1927: 119, fig. 42 h-1.

Piromis eruca; Day, 1973: 108.

OCORRÊNCIA - Estação 2, em fundos de silte grosso.

DISTRIBUIÇÃO - Atlântico norte; do Canal da Mancha ao sul da França; Mediterrâneo.

\section{OPHELIIDAE}

Ophelina aulogaster (Rathke, 1843)

Ammotrypane aulogaster Rathke, 1843: 188, est. 10 fig. 1-3; Fauvel, 1927: 133, fig. 47 a-e; Hartman, 1969: 310, 3 figs.

OCORRÊNCIA - Estações 1 e 2, em fundos de silte grosso e médio. DISTRIBUIÇÃO - Sul do Alasca ao oeste do México.

\section{STERNAS PIDAE}

Sternaspis capillata Nonato, 1966

Sternaspis capillata Nonato, 1966: 79-84, figs. 1-9; Nonato \& Luna, 1970b: 94; Nonato, 1981: 188-189.

OCORRÊNCIA - Estação 2, em fundos de silte grosso e médio. DISTRIBUIÇÄO - Brasil: costa nordeste, Sergipe e sul, Santa Catarina.

\section{CAPITEI LIDAE}

Nonatus longilineus Amaral, 1980

Nonatus longilineus Amaral, 1980b: 103, fig. 2.1-3. OCORRÊNCIA - Dragagem.

DISTRIBUIÇÃO - Brasil: costa sul, Santa Catarina.

Notomastus lobatus Hartman, 1947

Notomastus lobatus Hartman, 1947: 415-417, est. 51 figs. 1-5; Hartman, 
1969: 399, 5 figs.

OCORRÊNCIA - Estações 1 e 6, em fundos de silte médio e fino.

DISTRIBUIÇÃO - Golfo da Califórnia, México; Canal San Pedro. Brasil: costa sul-sudeste, São Paulo (Ubatuba) e Santa Catarina (Ilha de Santa Catarina).

\section{MALDANIDAE}

Euclymene coronata Verrill, 1900

Euclymene coronata Verrill, 1900: 655-656; Hartman, 1942b: 70-71, figs. 147-148.

OCORRÊNCIA - Estação 3, em fundos de areia média.

DISTRIBUIÇÃO - Bermudas.

Euclymene dalesi Mangum, 1966

Euclymene dalesi Mangum, 1966: 1-5, fig. 1 a-d.

OCORRÊNCLA - Nas 6 estações de coleta, em fundos de areia fina, areia muito fina e silte; dragagem.

DISTRIBUIÇÃO - Brasil: costa sudeste, São Paulo (Ubatuba).

\section{Euclymene droebachiensis Sars, 1872}

Euclymene droebachiensis Sars, 1872; Arwidsson, 1907: 218-226, est.

5 fig. 159-165; est. 9 figs. 313-314; est. 10 figs. 315-317; est. 12 figs. 368-370.

OCORRÊNCIA - Estações 3, 5 e 6, em fundos de areia fina, areia muito fina e silte fino.

DISTRIBUIÇĀO - Atlântico Norte.

\section{Heteroclymene robusta Arwidsson, 1907}

Heteroclymene robusta Arwidsson, 1907: 227-235, est. 5 figs. 171-178; est. 10 figs. 318-320; est. 12 figs. 371-372.

Clymene (Euclymene) robusta; Fauvel, 1927: 174-176, fig. 60 k-p.

OCORRÊNCIA - Estação 1, 4 e 6, em fundos de silte médio e fino; dragagem.

DISTRIBUIÇÃO - Atlântico (costa oeste da Irlanda) : Canal da Mancha, Mar do Norte.

\section{Lumbriclymene noemia Lana, 1983}

Lumbriclymene noemia Lana, 1983: 51-59, fig. 1.1-3; fig. 2.2 a-c; fig. 3. OCORRENCIA - Estações 2 e 3, em fundos de areia fina e sil te médio. DISTRIBUIÇÃO - Brasil: costa sudeste, São Paulo (Ubatuba).

\section{Maldane sarsi Malmgren, 1865}

Maldane sarsi Malmgren, 1865; Arwidsson, 1907: 251-261, est. 6 figs. 192-199; est. 10 figs. 333-338; Hartman. 1969: 461, 5 figs. OCORRÊNCIA - Estação 2, em fundos de sil te médio. DISTRIBUIÇÄO - Cosmopolita. 


\section{Praxillella affinis (Sars, 1872)}

Clymene affinis Sars, 1872: 412.

Praxillella affinis; Arwidsson, 1907: 177-183, est. 4 figs. 145-152;

est. 9 figs. 297-301; est. 12 figs. 364-366.

Clymene (Praxillella) affinis; Fauvel, 1927: 180, fig. 62 f-1.

Praxillella cf. affinis; Day, 1967b: 644-645, fig. 30.7 m-p.

OCORRËNCIA - Estação 1, em fundos de silte médio.

DISTRIBUIÇÃO - Atlântico Norte (Noruega ao sul da Irlanda) e Sul (sul da África, Cabo Columbine); Japão.

\section{OWENIIDAE}

Owenia fusiformis delle Chiaje, 1844

Owenia fusiformis delle Chiaje, 1844: 31; Fauvel, 1927: 203, figs. 71 a-f; Day, 1967b: 649-651, fig. 31.1 e-j; Nonato \& Luna, 1970b: 94; Orensanz \& Gianuca, 1974: 20; Pullier \& Amoureux, 1979: 187; Nonato, 1981: 195.

OCORRÊNCIA - Estações 1, 2, 3 e 5, em fundos de areia fina, silte grosso e silte médio; dragagem.

DISTRIBUIÇĀO - Cosmopolita. No Brasil ocorre desde o nordeste (Alagoas) até o Rio Grande do Sul.

\section{PECTINARIIDAE}

Pectinaria (Pectinaria) laelia Nonato, 1981

Pectinaria (Pectinaria) laelia Nonato, 1981: 197-200, figs. 214-217.

OCORRÊNCIA - Estação 1, em fundos de silte médio; dragagem. DISTRIBUIÇĀO - Brasil: costa sudeste, Rio de Janeiro (Ilha Grande e Parati) e São Paulo (Ubatuba).

\section{AMPHARETIDAE}

Ampharete kerguelensis Mc Intosh, 1885

Ampharet kerguelensis Mc Intosh, 1885: 426, est. 47 fig. 10, est. 26A figs. 22-24; Hessle, 1917: 100; Hartman, 1966: 77, est. 25 figs. 3-5; Day, 1967b: 700, fig. 35.4 k-1.

OCORRENNCIA - Dragagem

DISTRIBUIÇÃO - Antártica, Georgia do sul, Ilha Kerguelen; Nova Zelândia; oeste da África tropical.

Amphicteis latibranchiata Nonato, 1981

Amphicteis latibranchiata Nonato, 1981: 200-202, figs. 218-219.

OCORRÊNCIA - estação 2, em fundos de silte médio; dragagem. DISTRIBUIÇÃO - Brasil: costa sudeste, Rio de لl laneiro (Ilha Grande) e Säo Paulo (Ubatuba). 
Isolda pulchella Fritz Müller, 1858

Isolda pulchella Fritz Müller, 1858: 218-219, est. 7 fig. 26-27; Day, 1967b: 691-692, fig. 35.1 k-n; Nonato, 1981: 202-203, fig. 200.

OCORRÊNCIA - Estação 4, fundos de areia média e muito fina.

DISTRIBUIÇÃO - África do Sul; Brasil: costa sul-sudeste do Rio de Janeiro a Santa Catarina.

\section{TEREBELUDAE}

Artacama benedeni Kinberg, 1866

Artacama benedeni Kinberg, 1866b: 346, Hessle, 1917: 195-196, fig. 50; Orensanz \& Gianuca, 1974: 21; Nonato, 1981: 205-207, fig. 221.

OCORRÊNCIA - Estação 2, em fundos de silte grosso.

DISTRIBUIÇÃO - Atlântico Sul: Uruguai: Brasil: costa sul-sudeste, do Rio de Janeiro ao Rio Grande do Sul.

\section{Lanice conchilega (Pallas, 1766)}

Nereis conchilega (Pallas, 1766): 131, est. 9 figs. 14-22.

Lanice conchilega; Fauvel, 1927: 255, fig. 88 a-h; Day, 1967b: 743, fig. $36.8 \mathrm{n}-\mathrm{r}$.

OCORRÊNCIA - Estação 5, em fundos de areia muito fina.

DISTRIBUIÇÃO - Atlântico (da Suécia ao Canal da Mancha) e oeste da África tropical; Mediterrâneo; Golfo Pérsico; sul da Califórnia.

\section{Pista herpini Fauvel, 1928}

Pista herpini Fauvel, 1928: 160, fig. 2 a-h; 1953: 427-428, fig. 220 a-h.

OCORRÊNCIA - Estações 1 e 5, em fundos de areia fina e silte médio; dragagem.

DISTRIBUIÇÃO - Golfo de Manaar, Pamban; Golfo Pérsico. Brasil: costa sudeste, São Paulo.

Polycirrus plumosus (Wollebaek, 1912)

Ereutho plumosa Wollebaek, 1912: 82, est. 21 figs. 1-4.

Polycirrus plumosus; Hessle, 1917: 224; Day, 1967b. 718, fig. 36.3 a-d.

Polycirrus cf. plumosus; Nonato, 1981: 213-214, figs. 224-226.

OCORRÊNCIA - Estação 1, em fundos de silte médio.

DISTRIBUIÇÃO - Noruega. Brasil: costa sudeste, Rio de Janeiro (Ilha Grande) e São Paulo (Ubatuba).

Polycirrus tenuisetis Langerhans, 1880

Polycirrus tenuisetis Langerhans, 1880: 110, est. 5 fig. 25; Fauvel, 1927: 283-284, fig. 98 m-n; Day, 1967b: 715, fig. 36.2 a-c.

OCORRENCIA - Dragagem.

DISTRIBUIÇÃO - Canal da Mancha; Atlântico (Madeira). 
Streblosoma bairdi (Malmgren, 1866)

Grymaea bairdi Malmgren, 1866: 388-389, est. 19 fig. 69.

Streblosoma bairdi; Fauve1, 1927: 275, fig. $96 \mathrm{f}-\mathrm{n}$.

Streblosoma cf. bairdi; Nonato \& Luna, 1970b: 96, figs. 89-91.

OCORRÊNCIA - Estações 2, 3, 4 e 5, em fundos de areia fina, areia muito fina e silte médio.

DISTRIBUIÇÃO - Atlântico Norte; Ártico; Atlântico Sul; Brasil: costa nordeste, Alagoas e Sergipe, sudeste, São Paulo.

\section{TRICHOBRANCHIDAE}

Terebellides anguicomus Fritz Müller, 1858

Terebellides anguicomus Fritz Müller, 1858: 218, est. 7 fig. 22; Hessle, 1917: 141, fig. 33; Nonato \& Luna, 1970b: 97; Nonato, 1981: 214-216.

OCORRÊNCIA - Estações 1, 2 e 3, em fundos de areia fina, silte grosso e silte médio; dragagem.

DISTRIBUIÇÃO - Brasil: costa nordeste, Sergipe e sul-sudeste, Rio de Janeiro (Ilha Grande), São Paulo (Ubatuba) e Santa Catarina.

Terebellides stroemi Sars, 1835

Terebellides stroemi Sars, 1835: 48; Hessle, 1917: 137; Fauvel, 1953: 291, fig. 100 i-q; Day, 1967b: 713, fig. $36.1 \mathrm{f}-\mathrm{j}$.

OCORRENCIA - Estações 1, 4 e 5, em fundos de areia muito fina, silte grosso e silte médio.

DISTRIBUIÇÃO - Cosmopolita. Brasil: costa sudeste.

\section{SABEULDAE}

\section{Chone filicaudata Southern, 1914}

Chone filicaudata Southern, 1914: 141, est. 14-15 fig. 32; Fauvel, 1927: 337, fig. 117 a-k; Day 1967b: 776-777, fig 37.6 p-w. OCORRENCIA - Estação 3, em fundos de areia média. DISTRIBUIÇÃO - Atlântico: Irlanda, Carolina do Norte.

\section{CONSIDERAÇÕES ZOOGEOGRÁFICAS}

A costa sudeste do Brasil constitui uma área de particular interesse biogeográfico, conforme referido por LANA (1987), por ser considerada uma zona de transição entre a fauna tropical ou caribe e a fauna temperada ou patagônica. Baseando-se no fato de espécies termófilas estarem gradualmente menos representadas até os estados de Santa Catarina e Rio Grande do Sul, e nas informações sobre as características oceanográficas, sedimentológicas e faunísticas da região entre o Rio Grande do Sul e o Rio da Prata, que funcionaria como uma barreira ecológica na distribuição dos organismos tropicais, PALÁCIO (1982) reconheceu ser a plata- 
forma continental entre o Espírito Santo e o Rio Grande do Sul, uma região de transição faunística e a designou como Província Paulista. Esta província, limitada ao norte pela Província do Caribe e, ao sul, pela Província Patagônica, conteria uma proporção considerável de espécie autóctones, mas também membros das faunas caribe e patogônicas, além de organismos circum-tropicais. A Província Paulista é limitada ao norte pela isócrima (temperaturas mais frias durante 30 dias consecutivos) de $23^{\circ} \mathrm{C}$, na altura do Estado do Espírito Santo, e ao sul, pela isoterma de $23^{\circ} \mathrm{C}$, na altura do Uruguai .

Entre as 105 espécies de anelídeos poliquetos identificadas, sete são consideradas novas para a ciência e pertencem aos gêneros: Linopherus (Amphinomidae), Ninoe (Lumbrineridae), Haploscoloplos e Scoloplos (Orbiniidae), Ophelina (Opheliidae), Heteroclymene e Praxillella (Maldanidae) e 20 constituem ocorrência nova para a costa brasileira: Eunoe nodosa, Pholoe cf. synophthalmica, Gyptis arenicolus, Neanthes arenaceodonta, Nephtys paradoxa, Mooreonuphis pallidulla, Onuphis dibranchiata, Augeneria tentaculata, Stauronereis annulata, Aricidea neosuecica, Pherusa laevis, Piromis eruca, Ophelina aulogaster, Euclymene coronata, Euclymene droebachiensis, Heteroclymene robusta, Praxillella affinis, Ampharete kerguelensis, Polycirrus tenuisetis e Chone filicaudata.

Um exame global da coleção permitiu identificar alguns padrões de distribuição geográfica, apresentados a seguir, juntamente com o número e a porcentagem de espécies incluídas em cada padrão estabelecido. Não foram consideradas nesta análise, as formas mantidas a nível genérico.

Padrões de Distribuição Geográfica

Espécies Restritas à Província Paulista

Espécies Anfi-Americanas (costa atlântica e pacífica)

Espécies Cosmopolitas

Espécies Anfi-Atlânticas (incluindo Mediterrâneo)

Espécies Comuns às Províncias Paulista e Patagônica

Espécies Restritas ao Atlântico Ocidental (Hemisfério norte e sul)

Espécies Circum-Tropicais

Espécies Comuns às Províncias Caribe e Paulista

Espećies Comuns às Províncias Paulista, Magelânica e Antártica.

Espécies Comuns ao Atlântico Ocidental e Índico

Espécies Anfi-Americanas; Japão

Espécies Anfi-Atlânticas; Japão
Número de Abundância

Espécies Relativa (\%)

24

25,5

18

19,0

16

17,0

15

16,0

7

7,4

4

4,3

2

2,0

2

2

2,0

2

2,0

1

1,0

1

1,0 
As espécies consideradas endêmicas para a costa sudeste do Brasil, constituíram o maior segmento da coleção examinada, compreendendo $25,5 \%$ do total de espécies. Este elevado grau de endemismo foi também verificado por LANA (1984) para as espécies de poliquetos errantes do litoral do estado do Paraná. Aquelas com distribuição em ambos os lados das Américas representaram uma proporção também elevada, 19\%. Excetuando-se Anaitides mucosa e Ophelina aulogaster, as demais espécies são típicas de águas tropicais ou temperadas quentes, provavelmente originárias de uma fauna única, comun ao Atlântico e Pacífico, separadas após a ligação das Américas, conforme mencionado po AMARAL (1977b) e LANA (1984).

Um significativo segmento da fauna regional é constituída por espécies consideradas cosmopolitas $(17 \%)$ como Diopatra cuprea, Laonice cirrata e Paraprionospio pinnata, e por espécies restritas ao atlântico ocidental e oriental $(16 \%)$.

As espécies comuns às províncias Paulista e Patogônica estiveram pouco representadas $(7,4 \%)$. Segundo LANA (1984) estas espécies estariam mais restritas, no Estado do Paraná, aos fundos mais frios da plataforma continetal (abaixo de $50 \mathrm{~m}$ ), tendendo a aumentar p. a a região sul do país, conforme constatado po ORENSANZ \& GIANUCA (1974) e diminuir para o norte, onde a influência da região das Antilhas e Golfo do México é consideravelmente maior (NONATO \& LUNA, 1970b).

Sternaspis capillata e Terebellides anguicomus foram as únicas espécies comuns e exclusivas das províncias Caribe e Paulista. A participação de formas circum-tropicais é também pouco significativa ( $2 \%$ do total).

A contribuição de espécies magelânicas e antárticas para a constituição da fauna da costa sudeste brasileira, representadas na região estudada por Augeneria tentaculata e Ampharete kerguelensis, é, em geral, pouco significativa como também verificado por ORENSANZ \& GIANUCA (1974), AMARAL (1977b), TEMPERINI (1981) e LANA (1984).

A fauna de anelídeos poliquetos dessa região de Ubatuba é formada predominantemente por espécies de águas quentes, espécies endêmicas e espécies comuns ao Pacífico americano, com marcante contribuição de espécies anfi-atlânticas principalmente de águas temperadas. A afinidade com espécies patagônicas e caribes é bem menor do que a observada por AMARAL (1977b), NONATO (1981) e LANA (1984) para a costa sudeste do Brasil.

\section{REFERENCIAS}

ABREU, J. 1978 - Ecologia e Distribuição dos Polychaeta e Mollusca na Enseada da Fortaleza. Tese de Doutorado, Universidade de São Paulo, Instituto Oceanográfico, $77 \mathrm{pp}$.

AMARAL, A.C.Z. 1977a - Um poliqueto endoparasita, Labrorostratus prolificus sp. n. em Nereideo. Bolm Inst. oceanogr., S. Paulo, 26: 285-292. 
AMARAL, A.C.Z. 1977b - Anelídeos Poliquetos do Infralitoral em duas Enseadas da Regiäo de Ubatuba - Aspectos Ecologicos. Tese de Doutorado, Universidade de São Paulo, Instituto Oceanográfico, $137 \mathrm{pp}$.

AMARAL, A.C.Z. 1979 - Ecologia e contribuiçåo dos anelfdeos poliquetos para a biomassa bêntica da zona das marés, no litoral norte do Estado de São Paulo. Bolm Inst. oceanogr., S. Paulo, 28 (1): 1-52.

AMARAL, A.C.Z. 1980a - Anelídeos poliquetos do infralitoral em duas enseadas da região de Ubatuba. II. Aspectos ecológicos. Bolm Inst. oceanogr., S. Paulo, 29 (1): $69-87$.

AMARAL, A.C.Z. 1980b - Breve caracterização dos gêneros da famflia Capitellidae Grube (Annelida, Polychaeta) e descrição de Nonatus longilineus gen. sp. nov. Bolm Inst. oceanogr., S. Paulo, 29 (1): 99-106.

AMARAL, A.C.Z. \& E.F. NONATO, 1982 - Anelfdeoas poliquetos da costa brasileira. Aphroditidae e Polynoidae. Braślia, Coordenaçäo Editoral/CNPq, 3: 46pp.

AMARAL, A.C.Z. \& E.F. NONATO, 1984 - Anelf́deos poliquetos da costa brasileira. Polyodontidae, Pholoidae, Sigalionidae e Eulepethidae, Brasilia, Coordenação Edito $\mathrm{ral} / \mathrm{CNPq}$, 4: 54pp.

AMARAL, A.C.Z.; E.F. NONATO, \& E.H. MORGADO, 1987 - Alterações na fauna de anelídeos poliquetos da Praia do Saco da Ribeira, Ubatuba - SP. Anais Simp6sio sobre Ecossistemas da Costa Sul e Sudeste Brasileira: Sfntese dos Conhecimentos, Acad. Ciênc., Sāo Paulo, 3: 244-157.

* ANNENKOVA, N.P. 1934 - Meeres - Paraoniden in fernen Osten der USSR. Akad, Nauk SSSR, C.R. (Doklady) ns. 4: 656-661.

AR WIDSSON, I. 1907 - Studien über die Skandinavischen and arktischen Maldaniden nebst ZUsammenstellung der übrigen bisher bekannten Arten dieser Familie. Zool. Jahrb., Suppl., 9: 308pp.

AUGEneR, H, 1918 - Polychaeta, Beitr. Kennt. Meeresfauna Westafr., 2 (2): 67-625.

AUGENER, H. 1933 - Polychaeten aus den zoologischen Museen von Leiden und Amsterdan. III. Zoöl. Meded. Leiden, 17: 283-410

BANSE, K. 1977 - Gymnonereidinae new subfamily: the Nereididae with bifid parapodial neurocirri. J. nat. Hist., 11: 609-628.

BANSE, K. \& K.D. HOBSON, 1968 - Benthic polychaetes from Puget Sound, Washington, with remarks on four other species. S miths, Inst., 125 (3667): 1-53.

BLANKENSTEYN, A. \& P.C. LANA, 1987 - Octobranchus longipes sp. N. (Trichobranchidae: Polychaeta) da costa sudeste do Brasil. Arq. Biol. Tecnol., 30 (4): 671-767. 
Vol. 6(3), 1989

BOLfVAR, G.A. 1986 - Padrões de Distribuição de Spionidae e Magelonidae (Annelida:

Pol ychaeta) do Litoral do Estado do Paraná. Dissertação de Mestrado, Universidade Federal do Paraná, Setor de Ciências Biológicas, 116pp.

BOLfVAR, G.A. \& P.C. LANA, 1986 - Magelonidae (Annelida, Polychaeta) do litoral sudeste do Brasil. Nerítica, Pontal do Sul, PR, 1 (3): 131-147.

BOLÍ VAR, G.A. \& P.C. LANA, 1988 - Padrōes de distribuição de Spionidae e Magelonidae (Annelida: Polychaeta) do litoral do Estado do Paraná. An. Sem. Reg. Ecolo, Såo Carlos, SP. 6: 247-267.

* BOSC, L.A.G. 1802 - Histoire naturelle des vers contenant leur description et leur moeurs avec figures desinées d'aprés nature, Paris, 1: 113-187.

* ClaPARÈdE, E. 1868 - Annélides Chétopodes du Golfe de Naples. Mém. Soc. Phys, Hist. nat., Genève, 19 (2): 313-514.

${ }^{*}$ CLAPARÈdE, E. 1869 - Les annélides chétopodes du Golfe de Naples. Second Partie. Mêm. Soc. Phys. Hist. nat., Genève, 20 (1): 1-225.

*CLAPARĖdE, E. 1870 - Les annélides chétopodes du Golfe de Naples. Mém. Soc. Phys, Hist. nato, Genève, 29 (2): 365-542.

DAY, J.H. 1967a - A monograph on the Polychaeta of southern Africa. Part 1. Errantia. London, British Museum Nat. Hist. Publ., (655): xxxviii + 878pp.

DAY, J.H. 1967b - A monograph on the Polychaeta of southern Africa. Part 2. Sedentaria London, Bristish Museum Nat. Histo Publo, (656): xvii + 419pp.

DAY, J.H. 1973 - New Polychaeta from Beaufort, with a key to all species recorded from North Carolina. Tech, Rep. Natn. mar. Fish. Serv,, Circ., (375): 1-140.

DAY, J. H。; J.G. FIELD, \& M. MONTGOMERY, 1971 - The use of numerical methods to determine the distribution of benthic fauna across the continental shelf of North Carolina, J. Anim. Ecol., 40: 93-126.

* DElle ChiAJE, S. 1828 - Memoria sulla storia e notomia degli animali senza vertebra del Regno di Napoli, Naples, 3: 1-232.

* DELle CHIAJE, S. 1841-44 - Descrizione e notomia degli animali invertebrati della Sicilia cateriore osservati vivi negli anni 1822-1830. Naples, 3.

EHLERS, E. 1887 - Report on the annelids of the dredging expedition of the U.S. coast survey steamer "Blake". Mem. Mus. comp. Zoolo, Harv., 15: 1-335.

* EHLERS, E. 1897 - Polychaeten. Hamburger Magalhaenischen Sammel reise. Hamburg, Friedrichsen \& Co., 148pp. 
* EHLERS, E. 1901 - Die Polychaeten des magellanischen und chilenischen Strandes. Fetshr. Ges, Wiss. Götingen, Math. - Phys。 Kl: 1-232.

* EISIG, H. 1914 - Zur Systematik, Anatomie und Morphologie der Ariciiden nebst Beitragen sur generalen Systematik. Mitt. Zool. Stn., Neapel, 21: 153-600.

* EliAsON, A. 1920 - Biologisch - faunistische Unitersuchungen aus dem Öresund. V-Polychaeta. Lunds Univ. Arsskr. Aud., 16 (6): 1-103.

* FABRICIUS, O. 1780 - Fauna Groenlandica, systematice sistens, Animalia Groenlan dice Occidentalis hactenus indagata, quoad nomen specificum, triviale, vernaculumque: synon yma auctorum plurium, descriptionem, locum, generationem, mores, usum, capturamque singuli; prount detegendi occasio fuit, maximaque parti secundum proprias observationes. Hafniae et Lipsiae, 452pp.

FAUCHALD, K. 1970 - Polychaetous annelids of the families Eunicidae, Lumbrineridae, Iphitimidae, Arabellidae, Lysaretidae and Dorvilleidae from Western Mexico. Allan Hancock Monogr. mar. Biole, (5): 1-335.

FAUCHALD, K。 1976 - Some Nephtyidae (Polychaeta) from Ubatuba, Brazil. Bull. So. Calif. Acad. Scio, 75 (1): 16-19.

FAUCHALD, K. 1977 - Polychaetes from intertidal areas in Panama, with a review of previous shallow-water records. S miths, Contr. Zool., 221: $81 \mathrm{pp}$.

FAUCHALD, K. 1982a - Two new species of Onuphis (Onuphidae: Polychaeta) from Uruguay. Proc. Biol. Soc. Wash., 95 (1): 203-209.

FAUCHALD, K. 1982b - Revision of Onuphis, Nothria, and Paradiopatra (Polychaeta: Onuphidae) based upon type material. Smiths. Contr. Zoolo, (356): 109pp.

FAUVEL, P. 1923 - Polychète errantes. Faune Fr., 5: 1488.

FAUVEL, P. 1927 - Polychète sedentaires. Addenda aux errantes, Archiannélides, Myzostomaires. Faune Fr., 16: 1-494.

FAUVEL, P. 1928 - Annélides Polychètes de l'Inde, I. Bull. Mus, Hist. Nat. Paris, 34: $90-96$.

FAUVEL, P. 1953 - Annelida, Polychaeta. The Fauna of Indias including Pakistan, Ceylon, Burma and Malaya. The Indian Press, Allahabad: 507pp.

FORNERIS, L. 1969 - Fauna do sublitoral da Ilha Anchieta $\left(23^{\circ} 32^{\circ} \mathrm{S}\right.$ - $\left.45^{\circ} 04^{\circ} \mathrm{W}\right)$. Ciênc. e Cult., Sảo Paulo, 21 (2): 449-450.

FOSTER, N.M. 1971 - Spionidae (Polychaeta) of the Gulf of Mexico and the Caribbean Sea. Studies on the Fauna of Curação and other Caribbean Islands, 36: 1-183. 
Vol. 6(3), 1989

* FRITZ MULLER, 1858 - Einiges über die Anneliden Fauna der Insel St. Catharina an der Brazilianischen Küste. Arch. Naturg., Berlin, 24: 211-220.

GARDINER, S.L. 1975 - Errant polychaete annelids from North Carolina. J. Elisha Mitchell Sci. Soc., 91 (3): 77-200.

GARDINER, S.L. \& WILSON, W. H. 1979 - New records of polychaete annelids from North Carolina with description of a new species of Sphaerosyllis. J. Elisha Mitchell Sci. Soc., 93 (4): 159-172.

* GRUBE, A.E. 1878 - Annulata Semperiana, Beiträge zur Kenntniss der anneliden fauna der Philippinen nach den von Herrn Prof. Semper mitgebrachten Sammlungen. Mém. Acad. Sci. S. Peterb., 25: 1-300.

HANSEN, G.A. 1882 - Recherches sur les annélides recueilleis par M. le professeur Eduard van Beneden pendant son voyage au Brésil et à La Plata. Mếm. Acad. R. S ci. Belg., Bruxelles, 44 (3): 1-29.

HARTMAN, O. 1940 - Polychaetous annelids. Pt. II. Chrysopetalidae to Goniadidae. Allan Hancock Pacif. Exped., 7 (3): 173-287.

HARTMAN, O. 1941 - Some contributions to the biology and life history of Spionidae from California. Allan Hancock Pacif. Exped., 7 (4): 289-324.

HARTMAN, O. 1942a - Report on the scientific results of the "Atlantis" expeditions to the West Indies under the joint auspices of the University of Havana and Harvard University. Mens Soc. cub. Hist. nato, 16 (2): 89-104.

HARTMAN, O. 1942b - A review of the types of polychaetous annelids at the Peabody Museum of Natural History, Yale University. Bull. Binghan oceanogr. Coll., 8: 1098.

HARTMAN, O. 1944 - Polychaetous annelids. Pt. V. Eunicea, Allan Hancock Pacif. Exped., 10 (1): 1-238.

HARTMAN, O. 1945 - The marine annelids of North Carolina. Bull. Duke Univ. mar. Stn., (2): 1-54.

HARTMAN, O 1947 - Polychaetous annelis. Pt. VII. Capitellidae. Allan Hancock Pacif。 Exped. 10 (4): 391-481.

HARTMAN, O. 1948 - The marine annelids erected by Kinberg with notes on some other types in the Swedish Museum. Arkiv. för Zoologi, 42A (1): 1-137.

HARTMAN, O. 1950 - Polychaetous annelids. Goniadidae, Glyceridae, Nephtyidae. Allan Hancock Pacif. Exped。, 15 (1): 1-182.

HARTMAN, O. 1951 - The littoral marine annelids of the Gulf of Mexico. Publs 
Inst. mar. Sci., Univ. Texas, 2: 7-127.

HARTMAN, O. 1953 - Non-pelagic polychaeta of the Swedish Antartic Expedition 1901-1903. Further Zoological Results of the Swedish Antar. Exped., 4 (11): $1-83$.

HARTMAN, O 1957 - Orbiniidae, Apistobranchidae, Paraonidae and Longonomidae. Allan Hancock Pacif. Exped., 15 (3) 211-393.

HARTMAN, O. 1959 - Catalogue of the polychaetous annelids of the world. Parts 1 and 2. Occ. Pap. Allan Hancock Fdn。, 23: 1-628.

HARTMAN, O 1961 - Polychaetous annelids from California, Allan Hancock Pacif. Exped., 25: 1-226.

HARTMAN, O. 1965a - Catalogue of the polychaetous annelids of the world. Occ. Pap. Allan Hancock Fdn., 23: 1-197.

HARTMAN, O 1965b - Deep-water polychaetous annelids off New England to Bermuda and other North Atlantic areas. Occ. Pap. Allan Hancock Fdn., 28: 1-378.

HARTMAN, O 1966 - Polychaeta Myzoztomidae and Sedentaria of Antarctica Antarctic Res. Ser., 7: 1-158.

HARTMAN, O 1968 - Atlas of the errantiate polychaetous annelids from California. Los Angeles, Allan Hancock Fdn., 828pp.

HARTMAN, O 1969 - Atlas of the sedentariate polychaetous annelids from California. Los Angeles, Allan Hancock Fdn., 812pp.

HARTMAN, O \& BARNARD, J.L. - The benthic fauna of the deep basins off southern California. Pt. II. Allan Hancock Pacif. Exped, 22 (2): 69-297.

HARTMAN-SCHRÖEDER, G. 1965 - Zur kenntnis der eulitoralen polychaeten fauna von Hawaii, Palmyra und Samoa. Abh. v. Verh. Naturwiss. Vereins Hamburg, N.F., 9: 81-161.

HESSLE, C. 1917 - Zur Kenntnis der terebellomorphen polychaeten.Z ool. Bidr. Uppsala, 5: $39-258$.

* HESSLE, C. 1925 - Einiges über die Hesioniden und die Stellung der Gattung Ancistrosyllis. Ark. Zool., 17 (10): 1-37.

IMAJIMA M. \& HARTMAN, O. 1964a - The polychaetous annelids from Japan. Pt. I. Occ. Pap. Allan Hancock Fdn., (26): 1-237.

IMAJIMA, M. \& HARTMAN, O. 1964b - The polychaetous annelids of Japan。 Pt. II. Occ Pap. Allan Hancock Fdn., (26): 239-452. 
Vol. 6(3), 1989

* JOHNSON, H.P. 1901 - The Polychaeta of the Puget sound region. Proc. Boston Soc. nato Histo, 29: 381-437.

* KEFERSTEIN, W. 1862 - Untersuchungen über niedere Seethiere。 Z. wiss. Zoolo, 12: $1-147$.

* KINBERG, J.G.H. 1865 - Annulata nova. Oefv. Vetensk. Akad. Stockholm, Förh, 21: $559-574$.

* KINBERG, J.G.H. 1866a - Annulata nova. Oefv. Vetensk. Akad. Stockholm, Förh, 22: $239-258$.

* KINBERG, J.G.H. 1866b - Annulata nova. Oefv. Vetensk. Akad. Stockholm, Förh, 23: $337-357$.

KIRKEGAARD, J॰B. 1959 - The polychaeta of West Africa Pt. I. Sedentary species. Atlantide Rep., (5): 7-117.

LA GRECA, M. 1946 - Studii sui policheti del Golfo di Napoli Staz. Zool. Napoli Publ., 20 (3): 270-280.

LANA, P.C. 1981 - Padröes de Distribuição e Diversidade Específica de Anelídeos Poliquetos na Região de Ubatuba. Estado de Sảo Paulo. Dissertação de Mestrado, Universidade de São Paulo, Instituto Oceanográfico, 111pp.

LANA, P.C. 1983 - Lumbriclymene noemia sp. n. (Maldanidae, Polychaeta) da costa sudeste do Brasil, com uma sinopse do gênero. Arq. Biol. Tecnol., 26 (1): 51-59.

LANA, P.C. 1984 - Annelídeos Poliquetos Errantes do Litoral do Estado do Parană. Tese de Doutorado, Universidade de Så̉o Paulo, Instituto Oceanogråfico, 275pp.

LANA, P.C. 1986 - Nephtyidae (Annelida, Polychaeta) do litoral do Estado do Paraná (Brasil). Nerítica, Pontal do Sul, PR, 1 (1): 135-154.

LANA. P.C. 1987 - Padrōes de distribuiçåo Geogråfica dos poliquetos errantes (Annelida: Polychaeta) do Estado do Paraná. Ciênc. e Cult., 39 (11): 1060-1063.

LANA. P.C. \& H.H. SOVIERZOSKI, 1987 - Neanthes bruaca sp. n. e Nereis broa sp. n. (Nereididae: Polychaeta) da costa sudeste do Brasil. Arq. Biol. tecnolo, 30 (4): $677-688$.

* LANGERhANS, P. 1880-81 - Die Würmfauna von Madeira • Pt. 2. Z. wiss Zool., 33: $267-316 ; 34: 87-143$.

* LEIDY, J. 1855 - Contributions toward a knowledge of the marine invertebrates of the coasts of Rhode Island and New Jersey. J. Nat. Sci, Philad,, 3: 135-158.

* MC INTOSH, W.C. 1885 - Report on the Annelida Polychaeta collected by H.M.S. 
Revta bras. Zool.

Challenger during the years 1873-76. Rep. Scient. Results Challenger (Zool.), 12: $1-554$.

* MC INTOSH, W.C. 1910 - A monograph of the British Annelids. II Polychaeta. 2. Syllidae to Ariciidae. Ray Soc., London: 233-524.

* MALM, A.W. 1874 - Annulater i hafvet utmed sverges vestkust och omkring Göteborgs Vetensk. S amh. Handl., 14: 71-105.

* MAlmgren, A.J. 1865-66 - Nordiska Hafs - Annulater. Oefv, vetensk. Akad. S tockholm, Förh., 21: 51-110, 181-192; 22: 355-410.

* MALMGREN, A๘J 1867 - Annulata Polychaeta Spetsbergiae, Groenlandiae, Islandiae et Scandinaviae hactenus incognita, Oefv. Vetensk. Akad. Stockholm, Förh., 24: $127-235$.

MANGUM, C.P. 1966 - Two new specie of Clymenella (Polychaeta: Maldanidae) from Brazil. Postilla, (104): 1-10。

* MONRO, C.C.A. 1930 - Polychaete worms, “Discovery” Rep. 2: 1-222.

* MONRO, C.C.A. 1933 - The polychaeta Errantia colleted by Dr. C. Crossland at Colon in the Panama region and the Galapagos Islands during the expedition of the S.Y. “St. George”. Proc. Zool. Soc. London, 1933 (1): 1-96.

* MOORE. J.P. 1903 - Descriptions of two new species of Polychaeta from Woods Hole, Massachusetts. Proc. Acad. Nat. Sci. Philad., 55: 720-266.

* MOORE, J.P. 1906 - Additional new species of Polychaeta from the north Pacific. Proc. Acad. Nat. Sci. Philad., 58: 217-260.

* MOORE, J.P. 1911 - The Polychaetous Annelids dredged by the U.S.S. Albatross off the Coast of Southem California in 1904, III: Euphrosynidae to Goniadidae. Proc. Acad. Nat. Sci. Philad., 63: 234-318.

MORGADO, E.H. 1988 - Anelf́deos Poliquetos do Sublitoral da Regiåo de Ubatuba - SP, compreendida entre as Ilhas Anchieta e Vitória. Tese de Doutorado, Universidade Estadual de Campinas, Instituto de Biologia, 181pp.

MORGADO; E.H. \& A.C.Z. AMARAL, 1984 - Anelífeos poliquetos associados ao briozoário Schizoporella unicornis (Johnston). IV. Phyllodocidae e Hesionidae. Revta bras, Zoole, S Paulo, 2 (2): 49-54.

MORGADO, E.H. \& A.C.Z. AMARAL, 1986 - Onuphidae (Polychaeta) da regiẫo de Ubatuba, SP, com notas sobre distribuiçăo. Resumos XIV Congresso Brasileiro de Zoologia, Juiz de Fora, MG, 14: 23.

NONATO, E.F. 1958 - Sôbre duas arenícolas da costa brasileira (Annelida, Polychaeta). 
Vol. 6(3), 1989

Contrçōes Inst. oceanogr., Univ. S Paulo, sér. Oceanogr. Biol., (3): 1-7.

NONATO, E.F. 1963 - Poecilochaetus australis sp. nov• (Annelida, Polychaeta). Neotropica, 9 (28): 17-26.

NONATO, E.F. 1966 - Sternaspis capillata sp. n. (Annelida, Polychaeta). Bolm inst. oceanogr., S Paulo, 15 (1): 79-83.

NONATO, E.F. 1981 - Contribuição ao conhecimento dos Anelídeos Poliquetos da Plataforma Continetal Brasileira, entre Cabo Frio e o Arroio Chuf. Tese Livre Docência, Universidade de São Paulo, Instituto Oceanogråfico, 246pp.

NONATO, E.F. \& J.A.C. LUNA, 1970a - Sobre Alguns poliquetos de escama do nordeste do Brasil. Bolm Inst. oceanogr., S Paulo, 18 (1): 63-91.

NONATO, E.F. \& J.A.C. LUNA, 1970b - Anelf́deos poliquetos do nordeste do Brasil. I. poliquetos bentônicos da costa de Alagoas e Sergipe. Bolm Inst. oceanogr. S Paulo, 19: 57-130.

NONATO, E.F. \& A.CZ. AMARAL, 1976 - Distribuição dos anelídeos poliquetos na costa do Brasil. Resumos do III Simpósio Latino-americano sobre Oceanografia Biológica, San Salvador.

NONATO, E.F.; G.A. BOLfVAR, \& P.C. LANA, 1986 - Laonice branchiata, a new species of Spionidae (Annelida: Polychaeta) from the southeastern brasilian coast. Nerítica, Pontal do Sul, PR, 1 (3): 21-25.

* OERSTED, A.S. 1843 - Annulatorum danicorum conspectus. Kobenhaun, Fasc. I, Maricolae, 52pp.

ORENSANZ, J.M. 1973 - Los anelidos poliquetos de la Provincia Biogeografica Argentina. IV. Lumbrineridae. Physis, Sec. A., 32 (85); 343-393.

ORENSANZ, J.M. 1974a - Los anelidos poliquetos de la Provincia Biogeografica Argentina. V. Onuphidae. Physis, Sec. A., 33 (86): 75-122.

ORENSANZ, J.M. 1974b - Los anelidos poliquetos de la Provincia Biogeografica Argentina, VI. Arebellidae. Physis, Sec. A., 33 (87): 381-408.

ORENSANZ, J.M. 1975 - Los anelideos poliquetos de la Provincia Biogeografica Argentina. VII. Eunicidae Y Lysaretidae. Physis, Sec. A., 34 (88): 85-111.

ORENSANZ J.M. \& N.M. GIANUCA, 1974 - Contribuição ao conhecimento dos anelídeos poliquetos do Rio Grande do Sul. I Lista sistemática preliminar e descrição de três novas espécies. Comun. Mus. Ci. PUC-Rs。, 4: 1-37.

PALACIO, Fృ. 1982 - Revesión zoogeografica marina del Sur del Brasil. Bolm Inst. oceanogr., S Paulo, 31 (1): 69-92. 
* PA LlAS, P.S. 1766 - Miscellanea zoologica, etc.: Hagae Comitum: 72-145.

PETTIBONE, M.H. 1963 - Marine polychaete worm of the New England region. 1. Aphroditidae through Trochochaetidae. Bull. U.S. Nat. Mus., (277): 1-356.

PETTIBONE, M.H. 1966 - Revision of the Pilargidae (Annelida: Polychaeta), including descriptions of new species, and redescription of the pelagic Podarmus ploa Chaberlin (Polynoidae)。 Proc. U.S. Nat. Mus, 118 (3525): 155-207.

* RATHKE, H. 1843 - Beiträge zur Fauna Norwegens, Nova Acta Acad. Leop. Carol., Nat. Cur. Halle, 20: 1-264.

* RENIER, S.A. 1804 - Prospetto della Classe dei Vermi nominati e ordinati second il sistema di Bosc, Pådua: 15-17.

RULLIER, F. \& L. AMOUREUX, 1979 - Annélides polychètes. Campagne de la Calypso au large des côtes atlantiques de l'Amérique du Sud (1961-1962). Ann. Inst. oceanogr., 55, fasc. supplo: 10-206.

* SARS, M. 1835 - Beskrivelser og Iagttagelser over nogle moerkelige eller nye i Havet ven den Bergenske Kyst levende Dyr at Polypermes, Acalephernes, Radiaternes, Annelidernes classer, med en Kort oversight over de hid til at For flalte ren sammesteds fudne Arter og deres Forekommen, Bergen, 81pp.

* SARS, M. 1851 - Beretning on en i sommeren 1849 foretagen zoologisk Reise i Lofoten og Finmarken. Nyt Mag. Naturv. Oslo, 6: 121-211.

* SARS, M. 1861 - Om de ved Norges Kyster forekommende Arter of Annelides laegetn Polynoe. Vidensk. Selsk. Christiania Forh: 54-62.

* SARS, M. 1872 - Diagnoser af nye Annelider fra Christiania-fjorden。 Videsnk. Salsk. Christiania Forh: 406-417.

* SOUTHERN, R. 1914 - Archiannelida and Polychaeta (Clare Island Survey). Proc. R. Ir. Acad., 31 (47): 1-160.

* STIMPSON, W. 1856 - Descriptions of some new marine invertebrates. Proc. Acad. nat. Sci. Philad., 7: 385-395.

TEMPERINI, M.T. 1981 - Sistemática e Distribuição dos Poliquetos Errantes da Plataforma Continental entre as latitudes $23^{\circ} 05^{\prime} \mathrm{S}$ e $30^{\circ} 00^{\prime} \mathrm{S}$. Dissertação de Mestrado, Universidade de São Paulo, Instituto Oceanográfico, $89 \mathrm{pp}$.

* TREAdWELl, A.L. 1901 - The Polychaetous annelids of Porto Rico. Bull. U.S. Fish Comunn., 20: 181-210.

* TREADWElL. A.L. 1906 - Polychaetous annelids of the Hawaiian Islands, collected by the steamer Albatross in 1902. Bull. U.S. Fish. Comunn., 23: 1145-1181. 
Vol. 6(3), 1989

* VERrill, A.E. 1900 - Additions to the Turbellaria, Nemertinea and Annelida of the Bermudas, with revisions of some New England genera and species. Trans. Conn. Acad. Arts. Sci., 10: 595-671.

* WEBSTER, H.E. 1879 - Annelida chaetopoda of the Virginian coast. Trans, Albany Inst., N.Y., 9: 202-269.

* Willey, A. 1905 - Report on the Polychaeta collected by Professor Herdman, at Ceylon in 1902. Ceylon Pearl Oyster Fisheries, Suppl. Rep。, pt。 4: 243-324.

* WOllebAEK, A. 1912 - Nordeuropaeiske Annulater Polychaeta. I. Ammocharidae, Amphictenidae, Ampharetidae, Terebellidae og serpulidae. Skr. Vidensk Selsk. Christiania (Mat-1naturv. Klo), 1911 (18): 1-144.

* Referências nåo consultadas 